\title{
A simple fabrication of PVA-ZnS composite films with superior photocatalytic performance: Enhanced luminescence property, morphology and thermal stability
}

Mohammad Mizanur Rahman Khan, ${ }^{*},+,+$ Subrata Pal ${ }^{\dagger}$, Md. Mainul Hoque, ${ }^{\dagger}$ Md.

Rashedul Alam, $†$ Muhammad Younus ${ }^{\dagger}$ and Hisatoshi Kobayashi

†Department of Chemistry, Shahjalal University of Science and Technology, Sylhet-

3114, Bangladesh

International Center for Materials Nanoarchitectonics, National Institute for Materials

Science (NIMS), 1-2-1, Sengen, Tsukuba, Ibaraki 305-0047, Japan

(S) Supporting Information

KEYWORDS. PVA-ZnS composite films, Photoluminescence property, Optical transparency, Morphology, Thermal stability, Photocatalytic activity 


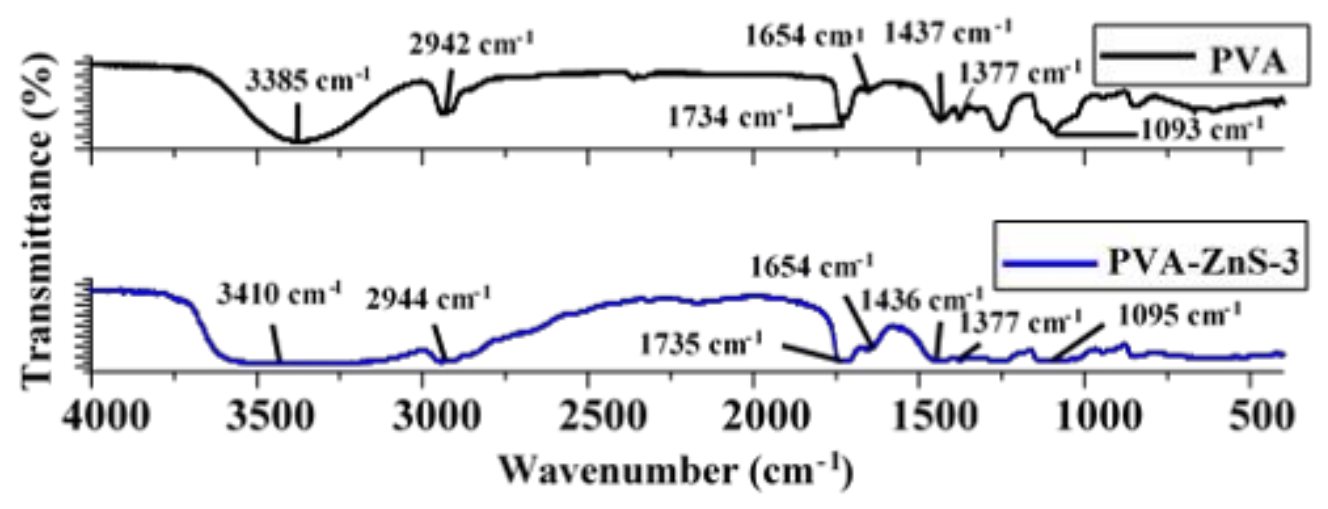

Figure S1. FTIR spectra of PVA and PVA-ZnS-3 composite films with detailed labelling of the peaks.

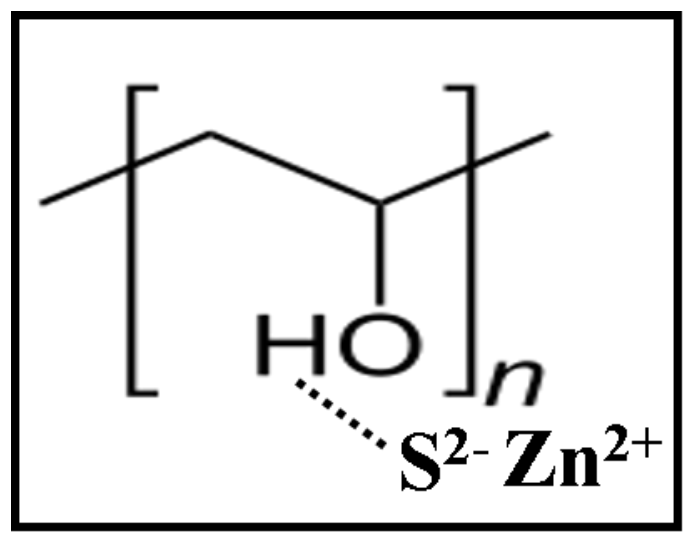

Figure S2. Possible hydrogen bonding between $\mathrm{ZnS}$ and PVA. The broken line indicates the hydrogen bond. 

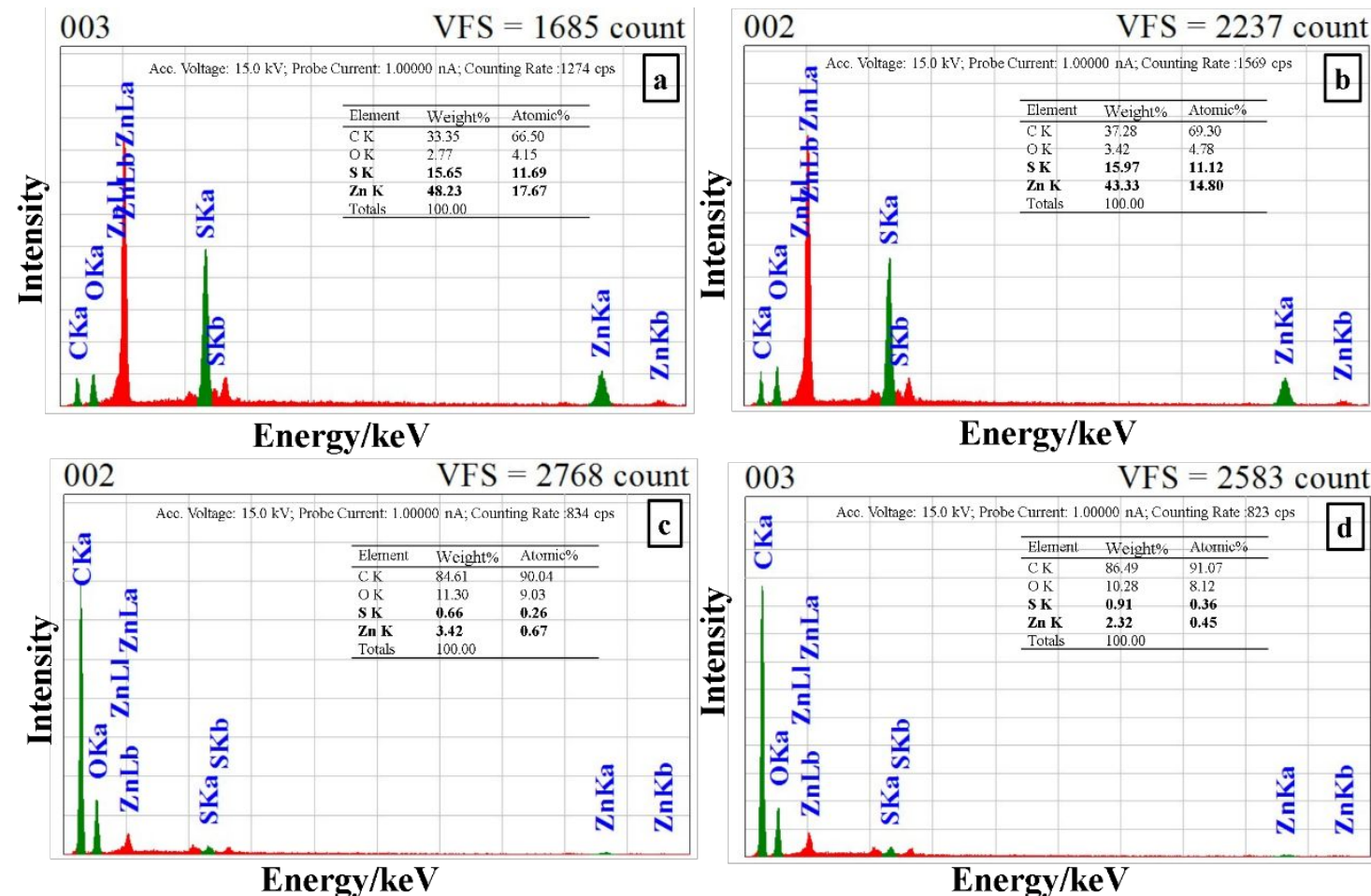

Energy/keV

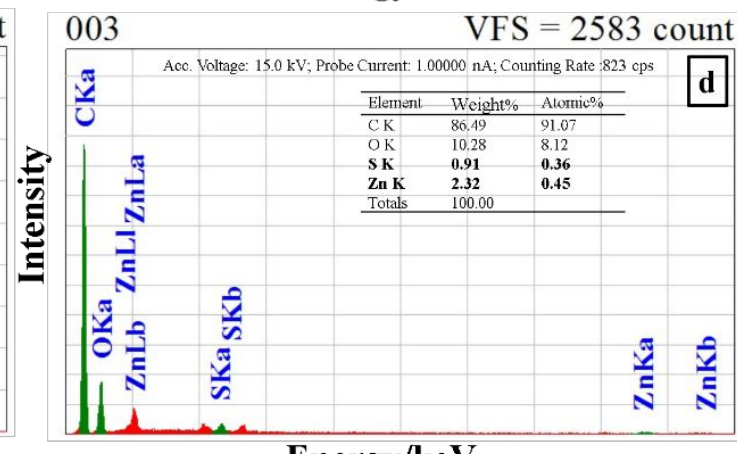

Energy/keV

Figure S3. EDX spectra of a) PVA-ZnS-2, b) PVA-ZnS-3, c) PVA-ZnS-4, and d) PVAZnS-5 composite films. 


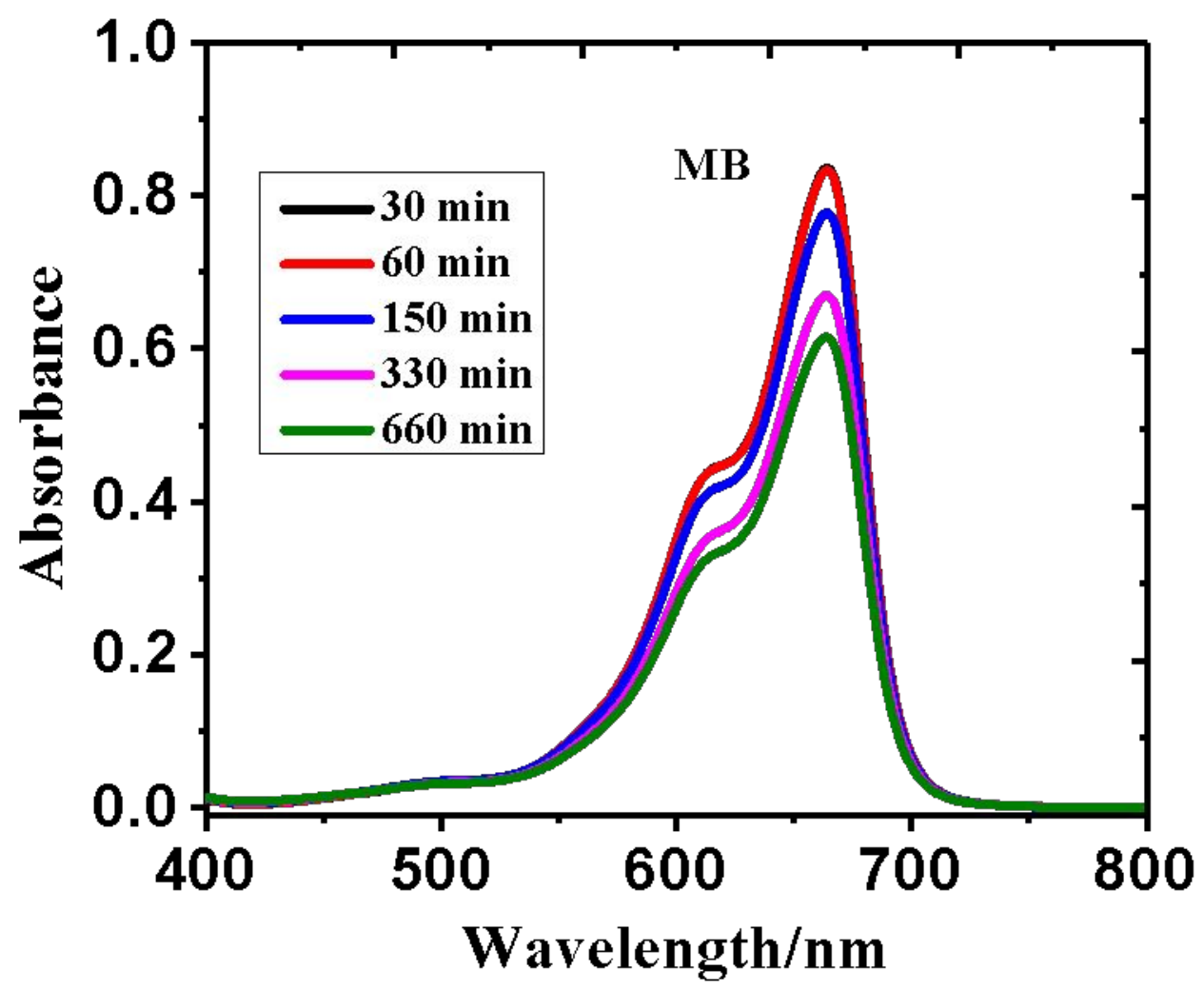

Figure S4. Changes in the UV-Vis absorption spectra of MB aqueous solution under sunlight irradiation. 


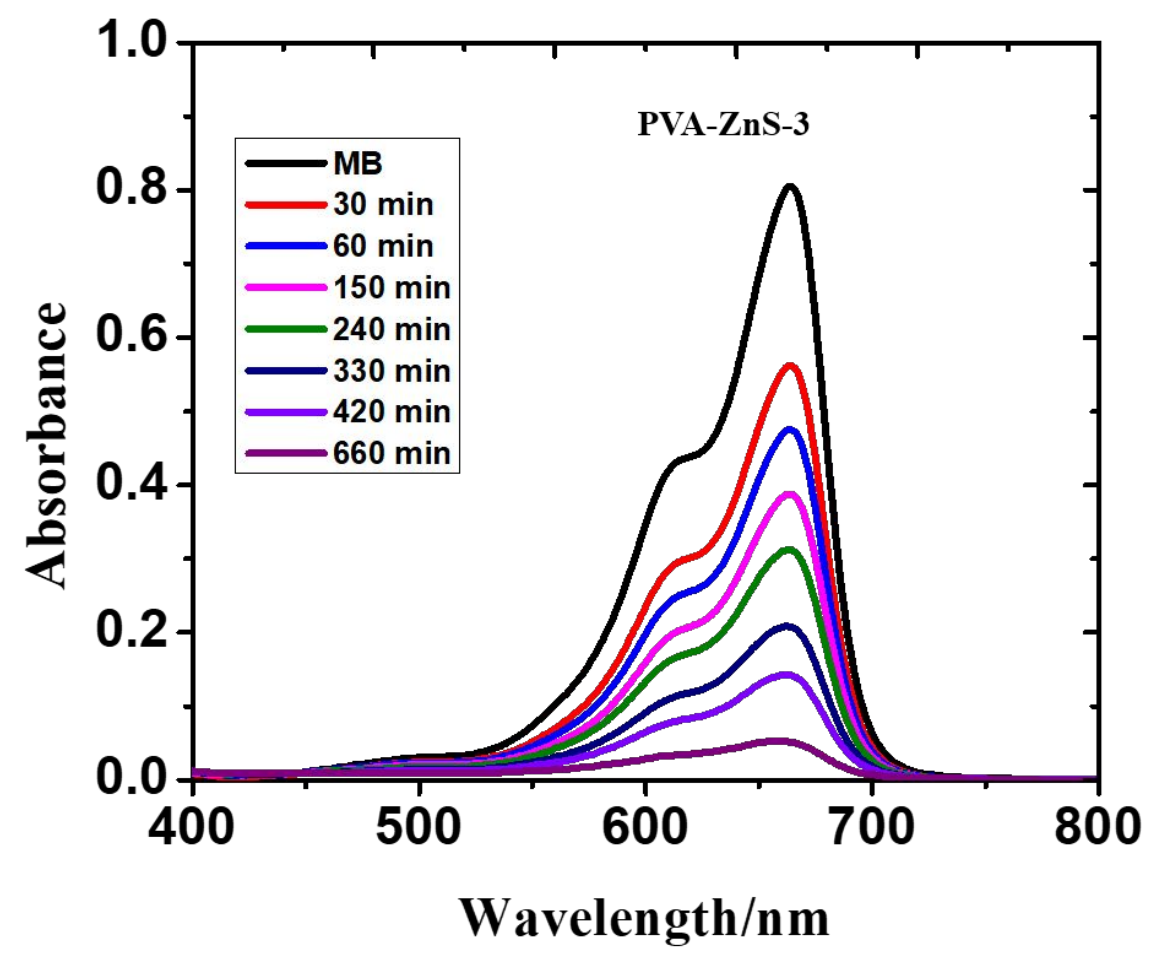

Figure S5. Changes in the UV-Vis absorption spectra of MB aqueous solution in the presence of PVA-ZnS composite films. The samples were reused ( 3 times) to check the photocatalytic performance. 

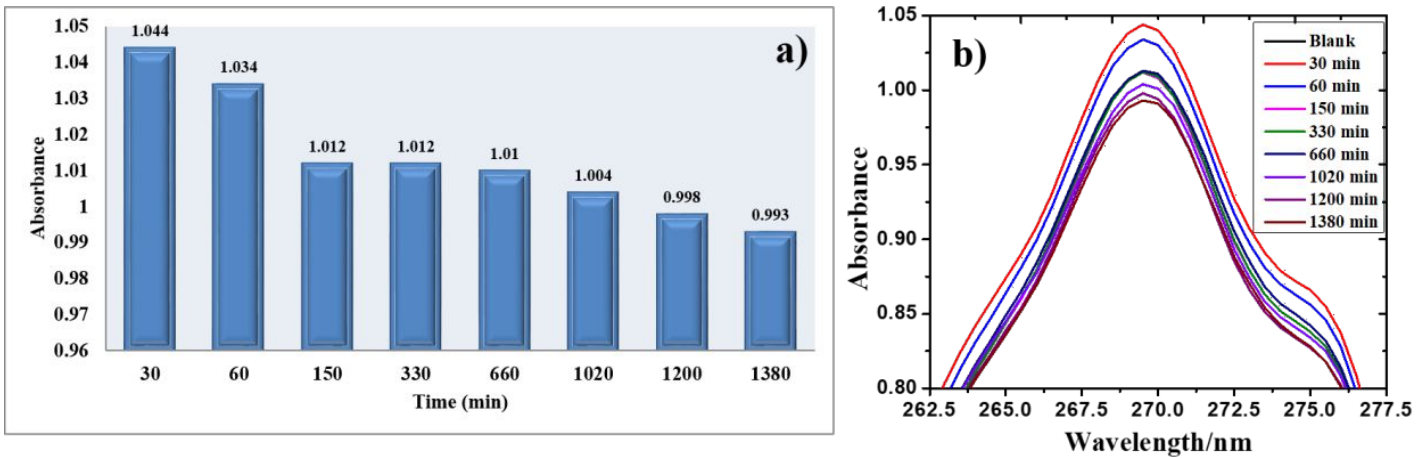

Figure S6. a) Plot of the absorbance versus time for the phototcatalytic degradation of phenol. b) Changes in the UV-Vis absorption spectra of phenol aqueous solution in the presence of PVA-ZnS composite films. 